médecine/sciences $1988 ; 4: 658-9$

corps anti-CD4. Ceux-ci bloquent la sélection positive et on ne retrouve pas de clones $\mathrm{CD}^{+}, \mathrm{CD}^{-}$; ils bloquent également la sélection négative... y compris des clones CD4$\mathrm{CD}^{+}[4,5]$.

Les expériences de Kisielow et al. [6] indiquent enfin que la sélection (au moins la sélection négative, mais aussi, évidemment, la sélection positive si elle est antérieure) se fait au niveau des précurseurs $\mathrm{CD} 4^{+}, \mathrm{CD}^{+}$: des souris transgéniques pour les gènes d'un récepteur $\alpha \beta$ spécifique de l'antigène HY (exprimé chez les mâles) présenté dans le contexte de molécules du $\mathrm{CMH}$ de classe I ne possèdent, chez les animaux mâles ayant les molécules du CMH pour lesquelles est restreint le récepteur transgénique, ni les cellules autoréactives matures $\mathrm{CD}^{-} \mathrm{CD}^{+}$ni les cellules précurseurs $\mathrm{CD}^{+} \mathrm{CD}^{+}$, alors que, chez les femelles (n'exprimant pas HY), ces cellules immatures $\mathrm{CD}^{+} \mathrm{CD}^{+}$exprimant le transgène sont nombreuses. Ainsi, pas à pas, grâce à l'éventail entier des techniques de recombinants d'ADN et d'immunologie, élucide-t-on en cette deuxième moitié des années 80 les bases moléculaires du système immunitaire et de son aptitude à différencier le soi du non-soi [7].

A. $\mathbf{K}$.

1. Hung Sia Teh, Kisielow P, Scott B, et al. Thymic major histocompatibility complex antigens and the $\alpha \beta$ T-cell receptor determine the CD4/CD8 phenotype of T cells. Nature $1988 ; 335: 229-33$.

2. Sha WC, Nelson CA, Newberry RD, Kranz DM, Russel JH, Loh DY. Selective expression of an antigen receptor on CD8bearing $T$ lymphocytes in transgenic mice. Nature 1988; 355 : 271-4.

3. Janeway CA. T-cell development : accessories or coreceptors. Nature 1988 ; 335 : 208-10. 4. Fowlkes BJ, Schwartz PH, Pardoll DM. Deletion of self-reactive thymocytes accurs at a CD4 ${ }^{+} \mathrm{CD}^{+}$precursor stage. Nature 1988 ; 334: 620-3.

5. MacDonald HP, Hengartner H, Pedrazzini $T$. Intrathymic deletion of self-reactive cells prevented by neonatal anti-CD4 antibody treatment. Nature 1988 ; 335 : 174-6.

6. Kisielow $\mathrm{P}, \mathrm{Blüthmann} \mathrm{H}$, Staerz VD, et al. Tolerance in T-cell-receptor transgenic mice involves deletion of nonmature $\mathrm{CD}^{+}, \mathrm{CD}^{+}$ thymocytes. Nature $1988 ; 333: 742$

7. Kourilsky P, Claverie J-M. Le modèle du soi peptidique. médecine/sciences $1988 ; 4$ :

\title{
Des modèles animaux pour l'étude du SIDA
}

L'absence de modèle animal d'une maladie, notamment d'une maladie infectieuse, constitue un considérable handicap pour l'étude physiopathologique de l'affection et pour les essais thérapeutiques. Les chimpanzés sont sensibles à l'infection par les virus du SIDA, mais développent une maladie inapparente. De plus, ils sont rares, chers, et leur espèce est considérée comme menacée de disparition. De nombreuses tentatives ont donc été entreprises de développer un modèle animal plus accessible.

L'une des tentatives les plus folles, voire les plus inquiétantes, a été récemment rapportée par l'équipe de Frederickson (NCI, Bethesda MD, USA) lors d'un récent colloque du Cold Spring Harbor laboratory, près de New York. Il s'agit de créer des animaux transgéniques ayant intégré la totalité du génome viral de HIV-l. Les souris ainsi produites développent au $10^{\mathrm{e}}$ jour de vie une maladie caractérisée par un retard de croissance, une infiltration lymphoïde périvasculaire dans le poumon, des signes cutanés évoquant un psoriasis, des adénopathies et une réponse anormale des lymphocytes $T$ aux mitogènes. Du virus infectieux est retrouvé dans la rate et les ganglions. Les animaux meurent aux environs du $30^{\mathrm{e}}$ jour. Les souris ayant une tendance difficilement contrariable à s'échapper, ce sont de véritables quartiers de haute sécurité comportant trois lignes indépendantes de protection contre la fuite des animaux qui ont dû être construits, afin d'éviter la dissémination de ces petites bêtes produisant du virus HIV!

D'autres animaux transgéniques exprimant la molécule humaine CD4, récepteur des virus HIV, ont été créés dans l'espoir - déçu pour l'instant - que cela suffirait à permettre d'infecter ces animaux par HIV. En fait, le virus est bien intégré dans les cellules de souris, mais il ne s'y réplique pas, ce qui semble indiquer qu'existe une barrière d'espèce intracellulaire au cycle viral de HIV.

Très récemment a été développé un modèle tout différent et qui pourrait être promis à un avenir extrêmement important : il s'agit de construire des chimères de souris possédant de manière stable les cellules immunitaires de l'espèce humaine. Ceci est envisageable grâce à la mutation SCID (severe combined immunodeficiency). Les souris homozygotes pour l'allèle autosomique scid, d'apparition spontanée, ont un déficit immunitaire touchant les cellules $B$ et les cellules $T$. L'anomalie ne porte pas sur les autres lignées hématopoïétiques ; elle semble intéresser, spécifiquement, le processus de recombinaison des segments constituant les gènes d'immunoglobuline et des récepteurs pour l'antigène des lymphocytes T. Ce serait le système de la «recombinase» qui serait anormal : capable d'entraîner la coupure de l'ADN au niveau des sites corrects, il ne catalyserait pas la réunion normale des segments, ce qui provoque des délétions de régions entières des gènes d'immunoglobuline et des récepteurs $T$. Du fait du déficit immunitaire, les souris scid/scid ne rejettent pas les xénogreffes (greffes en provenance d'autres espèces). D'autre part, des cellules du système immunitaire humain fœtal greffées à ces souris développent une tolérance immunitaire vis-à-vis des tissus de souris, si bien qu'il n'y a pas non plus de réaction du greffon contre l'hôte[l, 2]. Lorsque du thymus, du tissu ganglionnaire et du foie foetaux sont greffés à ces souris, on observe l'apparition de cellules $\mathrm{T}$ et d'anticorps humains durant plusieurs semaines (7 semaines en moyenne, 
parfois jusqu' à 20 semaines). L'état immunologique précis des souris greffées et les paramètres intervenant dans la variabilité de la durée de persistance des cellules humaines restent à explorer. Une autre équipe a démontré que des lymphocytes humains circulants normaux, injectés par voie intrapéritonéale aux souris scid/scid, permettaient également une reconstitution immunitaire partielle au prix d'une réaction du greffon contre l'hôte faible ou modérée [3]. Comme dans l'expérience précédente, les cellules humaines survivent plusieurs mois. Quoique des études complémentaires soient indispensables pour préciser les caractéristiques de ces chimères homme-souris, il apparaît d'ores et déjà qu'un tel modèle murin possédant des cellules humaines CD4 (+) est extrêmement prometteur pour l'étude de HIV.

Dernier modèle, imprévu, à avoir été proposé, enfin: le lapin [4]. Après création d'une péritonite aseptique, les lapins sont infectés par voie intrapéritonéale. Ils développent des anticorps anti-HIV qui persistent un an après l'infection et le virus peut être retrouvé dans les macrophages de l'animal qui ne présente cependant aucun signe de déficit immunitaire. Ainsi, alors même que les perspectives thérapeutiques de la maladie ne sont pas réjouissantes, se mettent au moins en place une série de modèles qui permettront probablement de tester plus facilement et plus rapidement les vaccins et les médicaments nouveaux.

\section{A. $\mathbf{K}$.}

1. McCune JM, Namikawa R, Kaneshima H, Schultz LD, Lieberman M, Weissman IL. The scid-hu mouse: murine model for the analysis of human hemato-lymphoid differentiation and function. Science $1988 ; 241$ : 1632-9.

2. Yancopoulos GD, Alt F. Reconstruction of an immune system. Science 1988; 241 : 15813

3. Mosier DE, Culizia RJ, BairdSM, Wilson DB. Transfer of a functional human immune systema to mice with severe combined immunodeficiency. Nature 1988; 355 : 256-9.

4. Filice G, Cereda PM, Varnier OE. Infection of rabbits with human immunodeficiency virus. Nature $1988 ; 355: 365-8$.

$\mathrm{m} / \mathrm{s} n^{\circ} 10 \mathrm{vol} .4$, décembre 88

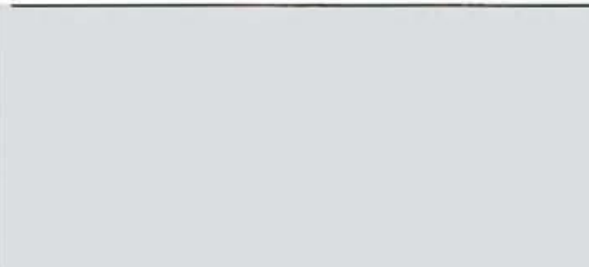

Des divas chez les poulets, qui chantent comme des cailleteaux. Nicole Le Douarin et son équipe viennent d'en créer [1] grâce aux greffes partielles de prosencéphale que nous décrivons ailleurs dans ce numéro de $\mathrm{m} / \mathrm{s}$ (mini-synthèse, page 648). Un des résultats les plus spectaculaires de ces manipulations de tissu nerveux embryonnaire est en effet l'altération du chant des jeunes poulets dans les semaines qui suivent l'éclosion. L'apparition précoce du chant peut être obtenue dans la semaine qui suit l'éclosion des poussins par stimulation à la testostérone. Alors, le jeune poulet produit normalement un son unique, fort et long d'une demi-seconde. La jeune caille («japonaise») produit plus généralement, de son côté, deux courtes «notes introductives » suivies d'un trille plus long et d'une fréquence irrégulière. Certains des jeunes poulets chez lesquels avaient été greffées des pièces de cerveau embryonnaire de cailles ont chanté... (presque) aussi mélodieusement que des cailleteaux. En fait, seuls les poulets chez lesquels le mésencéphale et le diencéphale de caille avaient été greffés - c'est-à-dire les régions du cerveau impliquées dans le chant ont joué les divas. Cette expérience démontre pour la première fois le transfert d'un comportement d'une espèce dans une autre, grâce à une greffe dans le système nerveux central, la réalisation du mythe des habitants de l'île du docteur Moreau! Les conséquences scientifiques de cette démonstration seront sans doute importantes. Quant aux retombées philosophiques...

[1. Balaban E, et al. Science 1988 ; 241 : 1339-42.]

Dolérance immunitaire aux antigènes HLA de la mère. Au cours du développement des lymphocytes $\mathrm{T}$ dans le thymus, les clones autoréactifs sont éliminés aboutissant à la tolérance de l'organisme aux antigènes du self, notamment à ses molécules du complexe majeur d'histocompatibilité (CMH). Une équipe néerlandaise [1] vient de montrer que, dans $50 \%$ des cas, il y avait aussi tolérance aux molécules du $\mathrm{CMH}$ de la mère non transmises à l'enfant. Il semble donc que, de même que le foetus peut immuniser la mère, la mère peut créer une tolérance immunitaire du foetus à certains de ses antigènes. Cette notion peut avoir d'importantes implications dans le choix d'un donneur de greffe.

[1. Class FHJ, et al. Science 1988; 241 : 815-7.]

Gaire la carte génétique de l'homme aussi simplement que celle de la drosophile. La drosophile a, pour le généticien, deux avantages essenticls sur l'homme: son génome est bien plus petit... et cela reste vrai. Sa descendance est nombreuse et sa reproduction rapide... et ces caractéristiques vont peut-être cesser d'être capitales. Non pas que l'on ait trouvé le moyen d'augmenter la taille et la vitesse de constitution des familles humaines, mais grâce à la PCR $\left(m / s n^{\circ} 8\right.$, vol. 4, p. 515) qui permet d'analyser individuellement les spermatozoïdes [1]. Un spermatozoïde est, après tout, la moitié d'un génome de descendant possible d'un homme... et il y en a quelques millions (de 20 à 200) par ml de sperme! Sur un seul prélèvement, il est ainsi possible de pratiquer des centaines d'analyses indépendantes permettant de calculer la distance génétique entre deux fragments d'ADN polymorphes avec une précision inférieure au centimorgan $(1 \mathrm{cM}=1 \%$ de recombinaison).

[1. Li H, et al. Nature 1988; 355 : 414-7.]
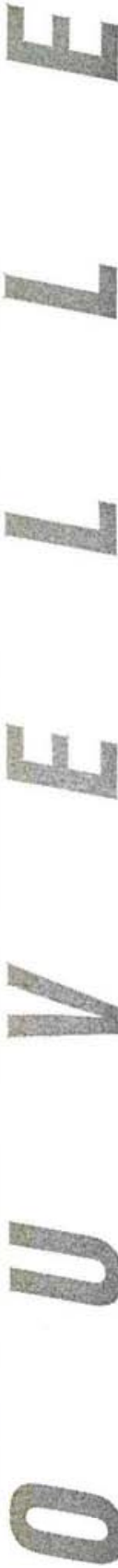